\title{
Association between visual impairment and sleep duration: analysis of the 2009 National Health Interview Survey (NHIS)
}

\author{
Alberto R Ramos ${ }^{1}$, Douglas M Wallace ${ }^{1}$, Natasha J Williams², David Warren Spence ${ }^{3}$, \\ Seithikurippu Ratnas Pandi-Perumal ${ }^{2}$, Ferdinand Zizi ${ }^{2}$ and Girardin Jean-Louis ${ }^{2^{*}}$
}

\begin{abstract}
Background: Visual impairment $(\mathrm{VI})$ is associated with increased mortality and health factors such as depression and cardiovascular disease. Epidemiologic studies consistently show associations between sleep duration with adverse health outcomes, but these have not systematically considered the influence of VI. The aim of this study was to ascertain the independent association between VI and sleep duration using the National Health Interview Survey (NHIS) data. We also examined whether race/ethnicity influenced these associations independently of sociodemographic and medical characteristics.
\end{abstract}

Methods: Our analysis was based on the 2009 NHIS, providing valid sleep and vision data for 29,815 participants. The NHIS is a cross-sectional household interview survey utilizing a multistage area probability design. Trained personnel from the US census bureau gathered data during face-to-face interview and obtained socio-demographic, self-reported habitual sleep duration and physician-diagnosed chronic conditions.

Results: The mean age of the sample was 48 years and $56 \%$ were female. Short sleep and long sleep durations were reported by $49 \%$ and $23 \%$ of the participants, respectively. Visual impairment was observed in $10 \%$. Multivariate-adjusted logistic regression models showed significant associations between $\mathrm{VI}$ and short sleep $(\mathrm{OR}=1.6,95 \% \mathrm{Cl}=1.5-1.9$ and long sleep durations ( $\mathrm{OR}=1.6,95 \% \mathrm{Cl}=1.3-1.9)$. These associations persisted in multivariate models stratified by race-ethnic groups.

Conclusion: Visual impairment was associated with both short and long sleep durations. Analysis of epidemiologic sleep data should consider visual impairment as an important factor likely to influence the amount of sleep experienced habitually.

Keywords: Visual Impairment, Sleep Duration, Race/ethnicity

\section{Background}

Visual impairment (VI) has been associated with increased mortality and adverse health factors such as depression and cardiovascular disease [1-3]. Few studies have observed that visually impaired individuals experience increased frequency of sleep symptoms and sleep/wake disorders [4-7]. Sleep difficulties, documented from clinical samples, include fragmented sleep, increased sleep

\footnotetext{
* Correspondence: Girardin.Jean-Louis@nyumc.org

${ }^{2}$ Center for Healthful Behavior Change (CHBC), Division of Health and Behavior, Department of Population Health, NYU Langone Medical Center, 227 East 30th Street, Floor \# 6-615, New York, NY 10016, USA

Full list of author information is available at the end of the article
}

onset latency, short sleep duration, and daytime naps [7]. Similar findings have been observed in population based studies from U.S. [6] and Swedish volunteers [8].

Epidemiological studies have linked sleep duration with increased mortality and cardio-metabolic disease [9]. Short sleep (<5-6 hours) and long sleep durations ( $>8-9$ hours) are associated to obesity, heart disease, stroke, diabetes mellitus, hypertension, and mood disturbances [9-11], but visual impairment have not been considered as an important factor in these studies. Within the purview of public health challenges associated with inadequate sleep, it has become important to assess the risk of medical comorbidities, such as visual impairment, 
that may be associated with sleeping below or above the modal population sleep time.

In light of the epidemiologic and clinical evidence that independently links adverse health outcomes with sleep duration and visual impairment; we sought to examine the associations between VI with sleep duration. Studies on the association between visual impairment and sleep [4-8] have been conducted in clinical samples or in elderly population-based studies, which limit their external validity. In contrast, this analysis was conducted among a representative sample of the US population with a diverse age, sex and ethnic background. Relevant to this point is evidence that race-ethnic disparities exist in disorders that may cause visual impairment (i.e. diabetic retinopathy, glaucoma) [12,13], sleep duration [14] and cardio-metabolic risk, $[15,16]$ such that individuals of the black race-ethnicity have a greater burden of visual impairment as well as an increased prevalence of both short and long sleep when compared with white counterparts $[17,18]$.

The aim of this study was to evaluate the associations between visual impairment and self-reported sleep duration in a representative sample of the US adult population, using data from the 2009 National Health Interview Survey (NHIS). Our hypothesis was that visual impairment is independently associated with short and long sleep durations. We also examined the associations among race/ethnicity, sleep duration and visual impairment, and whether these factors were independent of individual's sociodemographic and medical characteristics.

\section{Methods}

The study sample was derived from the 2009 NHIS data. The NHIS is conducted annually by the National Center for Health Statistics (NCHS), Centers for Disease Control and Prevention (CDC). The procedures involved in the NHIS and details concerning its sample design can be found in "NHIS Survey Description - 2009 Public Use Data Release" [19]. Briefly, the NHIS survey comprises a complex multistage area probability design that provides a representative sample of US households from all 50 states and the District of Columbia. In 2009, the interviewed sample consisted of 33,856 households, which yielded 88,446 persons in 34,640 families. There was a $65.4 \%$ response rate from the 34,616 eligible adults obtained from the Sample Adult questionnaire. Face-to-face interviews were conducted by trained interviewers from the US Census Bureau. The surveys were conducted using computer-assisted personal interviewing. Information was collected on socio-demographic data (age, sex, race/ethnicity, average family income, and education), health risks (smoking status and alcohol intake), and physician diagnosed chronic conditions or diseases such as hypertension, diabetes, coronary heart disease, cancer, kidney disease, stroke, and myocardial infarction.

The NHIS is a public data repository that is freely accessible online and can be downloaded and coded by an experienced bio-statistician using available coding and analytic instructions provided by the CDC. The research was conducted in accordance with the Declaration of Helsinki and was approved by the ethics committee [19].

\section{Visual impairment}

Participants reported visual impairment by answering a series of questions, as initiated by the Vision Health Initiative (VHI) branch of the CDC $[20,21]$. The interview documented whether a physician or other health professional ever informed participants that they had diabetic retinopathy, cataracts, glaucoma, or macular degeneration. Participants also reported if they were blind or unable to see, had trouble seeing even with corrective glasses or contacts, had a condition or health problem causing difficulty with seeing/vision, and the length of time they had a vision problem.

\section{Sleep duration}

Sleep duration was assessed by asking participants the following question: "On average, how many hours of sleep do you get in a 24-hour period?" Participants estimated habitual sleep duration using full hour units, i.e., 5 hours, 6 hours, and 7 hours. Sleep duration was categorized into 3 groups, short sleep duration ( $<6$ hours), long sleep duration ( $>8$ hours) with 6 to 8 hours of reported sleep used as the reference group [22].

\section{Assessment of covariates}

Chronic conditions documented in the interview included hypertension, heart disease and diabetes. Body mass index (BMI) was obtained by self-reported weight and height, and categorized based on established cut-offs (i.e., obesity consistent with an index $\geq 30 \mathrm{~kg} / \mathrm{m}^{2}$ ). Depressed mood was gathered by reporting "feeling sadness, hopelessness, worthlessness" in the previous 30 days before the face to face interviews [19].

\section{Statistical analysis}

Statistical analysis was carried out using the Statistical Package for the Social Sciences (SPSS) (version 18.0, SPSS Inc., Chicago, IL, USA) for windows. Chi-Square test was used to compare categorical data, while ANOVA was used to compare means for continuous variables. We performed logistic regression to evaluate the associations between visual impairment and short sleep and long sleep durations relative to the reference group. In our models, we adjusted effects of age, sex, race-ethnicity, income, BMI, as well as the presence of depressed moods, hypertension, diabetes and heart disease. 


\section{Results}

Our sample consisted of 29,815 volunteers aged 18-85 years. Table 1 shows descriptive information on demographics, medical factors, sleep duration and visual impairment. The mean age was 48 years, with $56 \%$ women, and more than $70 \%$ had a family income of less than $\$ 35,000$. Short sleep and long sleep durations were reported by $49 \%$ and $23 \%$ of the sample, respectively. Visual impairment was observed in $10 \%$.

Participants of White race-ethnicity constituted the majority of the sample. When compared to Whites, Blacks were younger, with a higher percentage of less than $\$ 35,000$ of family income. Blacks also had an elevated BMI, as well as an increased frequency of hypertension, diabetes mellitus, depressive symptoms, visual impairment and short and long sleep durations.

Evaluation of the covariates across the sleep duration groups showed that those with either short or long sleep duration had lower incomes and a greater frequency of hypertension, diabetes mellitus, and heart disease, when compared to participants with 6-8 hours of sleep (Table 2).

Sleep duration was associated with VI, in fully adjusted logistic regression models. Short sleep durations were associated with VI with stronger associations for long sleep in the unadjusted model. These associations were resilient to covariate adjustment: age, sex, income, and race-ethnicity, BMI, hypertension, diabetes, heart disease and depressive symptoms (Table 3). In stratified analysis, short sleep duration was associated to VI in Whites with a stronger association in Blacks. The association between long sleep and VI was mildly attenuated in Blacks when adjusting for main covariates (Table 4).

\section{Discussion}

We sought to ascertain associations between sleep duration and visual impairment (VI) in a representative sample of the US adult population. Participants who reported either short or long sleep durations had increased visual impairment. To our knowledge, this is the first study to document a relation between visual impairment and extremes of sleep duration, as conceived in the present analysis.

Consistent with prior studies, visual impairment had a U-shaped association with sleep duration $[6,9,18]$. In the present study, we observed associations between both short and long sleep durations and visual impairment after adjusting for depressed moods, low socioeconomic status and factors associated with sleep apnea (a known confounder for sleep duration) such as obesity, hypertension, diabetes mellitus, and heart disease. Our findings suggest that visual impairment could be a predictor of both short and long sleep durations. Even though causality cannot be determined, proposed mechanisms by which visual impairment could be associated with short and long sleep durations include shortened photoperiod and decreased circadian entrainment among participants with visual impairments [23].

Poor circadian entrainment and decreased exposure to daylight could lead to sleep fragmentation and early awakenings causing short sleep duration. In addition, poor circadian entrainment could lead to long sleep duration and or increased time in bed by altering the timing of sleep and rest-activity patterns $[4,7,8]$. In addition, long sleep might be a compensatory mechanism among those with fragmented sleep [24]. Poor sleep quality, increased sleep disturbances, and sleep apnea, a condition that leads to sleep fragmentation, are common in patients with visual impairment (i.e., glaucoma) [25-27]. The interplay among sleep duration, visual impairment and depression is also worth considering. In a study conducted among older volunteers [28] those with decreased ambient light perception, mainly explained by decreased visual acuity, had increased mood disturbances after accounting for sociodemographic, medical factors and sleep duration. Although

Table 1 Demographic, medical factors, sleep duration and visual impairment in the National Health Interview Survey

\begin{tabular}{|c|c|c|c|c|}
\hline (\%) & Total N= 29815 & Black $n=4407$ & White $N=25408$ & $P$ \\
\hline Age, years & $48 \pm 18$ & $46 \pm 17$ & $48 \pm 18$ & $<0.001$ \\
\hline Female sex & $56 \%$ & $61 \%$ & $55 \%$ & $<0.001$ \\
\hline Income, $<35,000$ & $77 \%$ & $84 \%$ & $76 \%$ & $<0.001$ \\
\hline $\mathrm{BMI}($ mean $\pm \mathrm{SD})$ & $27 \pm 6$ & $29 \pm 7$ & $27 \pm 6$ & $<0.001$ \\
\hline Diabetes & $8 \%$ & $12 \%$ & $8 \%$ & $<0.001$ \\
\hline Hypertension & $28 \%$ & $36 \%$ & $27 \%$ & $<0.001$ \\
\hline Heart disease & $8 \%$ & $6 \%$ & $9 \%$ & $<0.001$ \\
\hline Depressive symptoms & $26 \%$ & $29 \%$ & $26 \%$ & $<0.001$ \\
\hline Visual impairment & $10 \%$ & $11 \%$ & $10 \%$ & $<0.01$ \\
\hline Short sleep & $49 \%$ & $59 \%$ & $48 \%$ & $<0.001$ \\
\hline Long sleep & $23 \%$ & $31 \%$ & $22 \%$ & $<0.001$ \\
\hline
\end{tabular}

The P-value indicate differences between Black and White race-ethnic groups. 
Table 2 Demographic, medical factors and visual impairment across sleep duration categories: National Health Interview Survey 2009

\begin{tabular}{lcll}
\hline $\mathbf{N}=\mathbf{2 9 8 1 5}$ & $<\mathbf{6} \mathbf{h}$ & $\mathbf{6 - 8} \mathbf{~}$ & $\mathbf{>} \mathbf{8} \mathbf{~}$ \\
& $\mathbf{n = 8 , 4 5 4}$ & $\mathbf{n = 8 , 7 3 4}$ & $\mathbf{N}=\mathbf{2 , 5 9 3}$ \\
\hline & Demographic & & \\
Female, n (\%) & $4787(57)$ & $4737(54)$ & $1539(59)$ \\
Income, < 35,000, n (\%)* & $6377(75)$ & $6228(71)$ & $2261(87)$ \\
& Medical factors & & \\
Underweight, n (\%)* & $2821(33)$ & $3381(39)$ & $983(38)$ \\
Normal weight, n (\%)* & $2832(33)$ & $3132(36)$ & $869(34)$ \\
Obesity, n (\%) & $2801(33)$ & $2221(25)$ & $741(27)$ \\
Diabetes, n (\%)* & $768(9)$ & $540(6)$ & $332(13)$ \\
Hypertension, n (\%) & $2641(31)$ & $2090(24)$ & $938(36)$ \\
Heart Disease, n (\%)* & $757(9)$ & $617(7)$ & $325(13)$ \\
\hline *p<0.001. & & &
\end{tabular}

we did not obtain measures of visual acuity in our sample, it is plausible that decreased ambient light perception may adversely affect mood, potentially leading to extremes of sleep duration. The present study represents the first attempt to use a large, multi-ethnic representative sample of the U.S. population to assess the associations among sociodemographic variables, sleep duration and visual impairment.

However, the study has some limitations. One such limitation relates to the use of cross-sectional data, preventing inferences about causal relationships. Reverse causation is a possibility, provided that abnormal sleep habits/patterns are associated with diabetes, and cardiovascular disease, both of which are risk factors for ocular disease. It is plausible that abnormal sleep patterns may increase the risk of visual impairment by affecting systemic and ocular blood supplies, but additional research

Table 3 The Association between visual impairment and sleep duration in the National Health Interview Survey, 2009

\begin{tabular}{ccc}
\hline & \multicolumn{2}{c}{ Visual impairment } \\
\hline Short sleep, ${ }^{*}<\mathbf{6}$ hours & OR & $95 \%$ C.I \\
Model A & 1.8 & $1.6-2.0$ \\
Model B & 1.8 & $1.6-2.1$ \\
Model C & 1.6 & $1.5-1.9$ \\
Long sleep, ${ }^{*}>\mathbf{8}$ hours & & \\
Model A & 2.3 & $2.0-2.8$ \\
Model B & 1.8 & $1.5-2.1$ \\
Model C & 1.6 & $1.3-1.9$ \\
\hline
\end{tabular}

*6-8 hours of sleep was the reference group. Model A: Unadjusted.

Model B: Adjusted for age, sex, income, race-ethnicity.

Model C: Adjusted for Model B and BMI, hypertension, diabetes, heart disease, depression.
Table 4 The association between visual impairment and sleep duration stratified by race-ethnic groups

\begin{tabular}{|c|c|c|c|c|}
\hline \multirow{2}{*}{$\begin{array}{l}\text { Visual impairment } \\
\text { OR ( } 95 \% \text { C.I.) }\end{array}$} & \multicolumn{2}{|c|}{ White } & \multicolumn{2}{|c|}{ Black } \\
\hline & $<6$ hours & $>8$ hours & $<6$ hours & $>8$ hours \\
\hline Model A & $1.9(1.6-2.2)$ & $2.4(2.0-2.8)$ & $2.2(1.6-3.2)$ & $2.2(1.4-3.4)$ \\
\hline Moc & $1.8(1.6-2.1)$ & $1.8(1.5-2.1)$ & $2.2(1.5-3.1)$ & $1.8(1.1-2$ \\
\hline lodel C & $1.6(1.4-1.9)$ & $1.6(1.3-1.9)$ & $1.9(1.3-2.8)$ & $1.6(1.0-2$ \\
\hline
\end{tabular}

6-8 hours was the reference group.

Model A: Unadjusted.

Model B: Adjusted for age, sex, income.

Model C: Adjusted for Model B and BMI, hypertension, diabetes, heart

disease, depression.

studies are necessary. We did not obtain information on the visual acuity, physical activity, and medications that could affect sleep duration in our sample. Objective measures of sleep were not obtained (i.e. actigraphy and or polysomnography). In addition, measures of sleep quality (i.e. Pittsburgh Sleep Quality Index) were not collected in our sample. Self-reported sleep duration does not allow differentiating between total sleep time (TST) and time in bed (TIB). However, TIB and objective measures of TST are highly correlated, and studies that have discriminated between both measures have found similar associations with increased mortality $[29,30]$.

\section{Conclusion}

Visual impairment was associated with inadequate (short and long) sleep duration. Although prospective studies are needed to replicate the present findings, our study suggests that visual impairment should be considered as an important factor likely to influence interpretation of epidemiologic sleep data.

\section{Competing interest}

The authors report no competing interest. The authors alone are responsible for the content and writing of this manuscript.

Authors' contributions

Conceived and designed the study: GJL and FZ; Performed the study: ARR, NJW, and DMW; Analyzed the data: GJL and ARR; Contributed reagents/ materials/analysis tools: NJW, DMW; Helping to search the literature: ARR, DWS, and SRP; Wrote/contributed/critically reviewed the paper: ARR, NJW, DMW, DWS, SRP, FZ, and GJL; All authors read and approved the final submitted version of the manuscript.

Acknowledgement

The authors want to thank the participants for their time and contribution to this study. The project described was supported by Grant Number 1KL2TR000461 (ARR), R25HL105444, U54NS081765, and R01MD004113.

\section{Author details}

'Department of Neurology, Miller School of Medicine, University of Miami, Miami, FL, USA. ${ }^{2}$ Center for Healthful Behavior Change (CHBC), Division of Health and Behavior, Department of Population Health, NYU Langone Medical Center, 227 East 30th Street, Floor \# 6-615, New York, NY 10016, USA. ${ }^{3} 652$ Dufferin Street, Toronto, ON M6K 2B4, Canada.

Received: 10 April 2014 Accepted: 24 September 2014

Published: 1 October 2014 


\section{References}

1. Augustin A, Sahel JA, Bandello F, Dardennes R, Maurel F, Negrini C, Hieke K, Berdeaux G: Anxiety and depression prevalence rates in age-related macular degeneration. Invest Ophthalmol Vis Sci 2007, 48:1498-1503.

2. Karpa MJ, Mitchell P, Beath K, Rochtchina E, Cumming RG, Wang JJ: Direct and indirect effects of visual impairment on mortality risk in older persons. Arch Ophthalmol 2009, 127:1347-1353.

3. Lam BL, Lee DJ, Gomez-Marin O, Zheng DD, Caban AJ: Concurrent visual and hearing impairment and risk of mortality: the National Health Interview Survey. Arch Ophthalmol 2006, 124:95-101.

4. Lockley SW, Arendt J, Skene DJ: Visual impairment and circadian rhythm disorders. Dialogues Clin Neurosci 2007, 9:301-314.

5. Wee R, Van Gelder RN: Sleep disturbances in young subjects with visual dysfunction. Ophthalmology 2004, 111:297-302. discussion 02-3.

6. Zizi F, Jean-Louis G, Magai C, Greenidge KC, Wolintz AH, Heath-Phillip O: Sleep complaints and visual impairment among older Americans: a communitybased study. J Gerontol A Biol Sci Med Sci 2002, 57:M691-M694.

7. Lanzani MF, De Zavalia N, Fontana H, Sarmiento MI, Golombek D, Rosenstein RE: Alterations of locomotor activity rhythm and sleep parameters in patients with advanced glaucoma. Chronobiol Int 2012, 29:911-919.

8. Asplund R: Sleep, health and visual impairment in the elderly. Arch Gerontol Geriatr 2000, 30:7-15.

9. Cappuccio FP, Cooper D, D'elia L, Strazzullo P, Miller MA: Sleep duration predicts cardiovascular outcomes: a systematic review and meta-analysis of prospective studies. Eur Heart J 2011, 32:1484-1492.

10. Knutson KL: Sleep duration and cardiometabolic risk: a review of the epidemiologic evidence. Best Pract Res Clin Endocrinol Metab 2010, 24:731-743.

11. Kronholm E, Laatikainen T, Peltonen M, Sippola R, Partonen T: Self-reported sleep duration, all-cause mortality, cardiovascular mortality and morbidity in Finland. Sleep Med 2011, 12:215-221.

12. El-Gasim M, Munoz B, West SK, Scott AW: Discrepancies in the concordance of self-reported vision status and visual acuity in the Salisbury Eye Evaluation Study. Ophthalmology 2012, 119:106-111.

13. Leske MC, Connell AM, Wu SY, Nemesure B, Li X, Schachat A, Hennis A: Incidence of open-angle glaucoma: the Barbados Eye Studies. The Barbados Eye Studies Group. Arch Ophthalmol 2001, 119:89-95.

14. Nunes J, Jean-Louis G, Zizi F, Casimir GJ, von Gizycki H, Brown CD, McFarlane SI: Sleep duration among black and white Americans: results of the National Health Interview Survey. J Natl Med Assoc 2008, 100:317-322.

15. Mensah GA, Mokdad AH, Ford ES, Greenlund KJ, Croft JB: State of disparities in cardiovascular health in the United States. Circulation 2005, 111:1233-1241.

16. Campbell JA, Walker RJ, Smalls BL, Egede LE: Glucose control in diabetes: the impact of racial differences on monitoring and outcomes. Endocrine 2012, 42:471-482.

17. Hale L, Do DP: Racial differences in self-reports of sleep duration in a population-based study. Sleep 2007, 30:1096-1103.

18. Ram S, Seirawan H, Kumar SK, Clark GT: Prevalence and impact of sleep disorders and sleep habits in the United States. Sleep Breath 2010, 14:63-70.

19. Pleis JR, Ward BW, Lucas JW: Summary health statistics for U.S. adults: National Health Interview Survey. Vital Health Stat 10 2010, 249:1-207. Series 10, Data from the National Health Survey.

20. Mangione CM, Berry S, Spritzer K, Janz NK, Klein R, Owsley C, Lee PP: Identifying the content area for the 51-item National Eye Institute Visual Function Questionnaire: results from focus groups with visually impaired persons. Arch Ophthalmol 1998, 116:227-233.

21. Mangione CM, Lee PP, Pitts J, Gutierrez P, Berry S, Hays RD: Psychometric properties of the National Eye Institute Visual Function Questionnaire (NEI-VFQ). NEI-VFQ field test investigators. Arch Ophthalmol 1998, 116:1496-1504.

22. Zizi F, Pandey A, Murrray-Bachmann R, Vincent M, McFarlane S, Ogedegbe G, Jean-Louis G: Race/ethnicity, sleep duration, and diabetes mellitus: analysis of the National Health Interview Survey. Am J Med 2012, 125:162-167.

23. Grandner MA, Drummond SP: Who are the long sleepers? Towards an understanding of the mortality relationship. Sleep Med Rev 2007, 11:341-360.

24. Stepanski EJ: The effect of sleep fragmentation on daytime function. Sleep 2002, 25:268-276.
25. Bendel RE, Kaplan J, Heckman M, Fredrickson PA, Lin SC: Prevalence of glaucoma in patients with obstructive sleep apnoea-a cross-sectional case-series. Eye (Lond) 2008, 22:1105-1109.

26. Lin PW, Friedman M, Lin HC, Chang HW, Wilson M, Lin MC: Normal tension glaucoma in patients with obstructive sleep apnea/hypopnea syndrome. J Glaucoma 2011, 20:553-558.

27. Mojon DS, Hess CW, Goldblum D, Fleischhauer J, Koerner F, Bassetti C, Mathis J: High prevalence of glaucoma in patients with sleep apnea syndrome. Ophthalmology 1999, 106:1009-1012.

28. Jean-Louis G, Kripke D, Cohen C, Zizi F, Wolintz A: Associations of ambient illumination with mood: contribution of ophthalmic dysfunctions. Physiol Behav 2005, 84:479-487

29. Patel SR, Blackwell T, Ancoli-Israel S, Stone KL: Sleep characteristics of selfreported long sleepers. Sleep 2012, 35:641-648.

30. Youngstedt SD, Kripke DF: Long sleep and mortality: rationale for sleep restriction. Sleep Med Rev 2004, 8:159-174.

doi:10.1186/1471-2415-14-115

Cite this article as: Ramos et al: Association between visual impairment and sleep duration: analysis of the 2009 National Health Interview Survey (NHIS). BMC Ophthalmology 2014 14:115

\section{Submit your next manuscript to BioMed Central and take full advantage of:}

- Convenient online submission

- Thorough peer review

- No space constraints or color figure charges

- Immediate publication on acceptance

- Inclusion in PubMed, CAS, Scopus and Google Scholar

- Research which is freely available for redistribution
C Biomed Central 